\title{
A diameter bound for Sasaki manifolds
}

\author{
YASUFUMI NITTA
}

\begin{abstract}
In this paper we shall show that the diameter of a complete Sasaki manifold whose transverse Ricci curvature is bounded from below by a positive constant has a universal upper bound. This gives another proof of the result of Hasegawa and Seino in [9] which asserts that such manifolds are always compact with finite fundamental group.
\end{abstract}

Mathematics Subject Classification (2010): 53C55 (primary); 53D10 (secondary).

\section{Introduction}

A Sasaki manifold is a Riemannian manifold $(S, g)$ whose cone metric $\bar{g}=d r^{2}+$ $r^{2} g$ on $C(S)=S \times \mathbb{R}_{+}$is Kähler. Then Sasakian geometry sits naturally in two aspects of Kähler geometry. For one thing, $(S, g)$ is the base of the cone manifold $(C(S), \bar{g})$ which is a Kähler manifold. For another thing, any Sasaki manifold has a contact structure $(D, \eta, \xi)$, and it also has a 1-dimensional foliation $\mathcal{F}_{\xi}$, called the Reeb foliation, which admits a transverse Kähler metric $g^{T}$. Here, the Killing vector field $\xi$ is called the characteristic or Reeb vector field, the 1-form $\eta$ is called the contact 1-form, and the subbundle $D=\operatorname{ker} \eta$ is called the contact distribution ( $c f$. Section 2). For this reason, Sasaki manifolds are often described as odd dimensional counterparts of Kähler manifolds. Then it is a natural problem to investigate how restrictions on the curvature for the "Kähler structure" influence the topology of the manifold. For instance, by a result of Harada [7], for any compact regular Sasaki manifold $(S, g)$ such that $h>\kappa^{2}$ and the least upper bound of the sectional curvature is $1 / \kappa^{2}$, the fundamental group $\pi_{1}(S)$ is cyclic. Here $h=\inf \{H(X) ; X \in T S,|X|=1\}$ and $H(X)$ is the $\Phi$-holomorphic sectional curvature of $X$. (For the definition of $\Phi$-holomorphic sectional curvature, see [3].) Moreover, if additionally $S$ has minimal diameter $\pi$ then $S$ is isometric to the standard sphere ( $c f$. [8]). The classification of complete simply connected Sasaki manifolds with constant $\Phi$-holomorphic sectional curvature is obtained by 
Tanno $[16,17]$. An array of findings in this direction is described in the monograph of Blair [3].

In the present paper we shall approach the problem from the viewpoint of transverse geometry, and show a Myers' type theorem, as stated below. We say that the transverse Ricci curvature $\operatorname{Ric}^{T}$ is bounded from below if there exists a constant $\tau \in \mathbb{R}$ such that $\operatorname{Ric}^{T}(X, X) \geq \tau g^{T}(X, X)$ for each $X \in D$. We express the condition by $\operatorname{Ric}^{T} \geq \tau g^{T}$. Hasegawa and Seino showed in [9] that every complete Sasaki manifold with $\operatorname{Ric}^{T} \geq \tau g^{T}$ for some $\tau>0$ is compact with finite fundamental group. Here we shall show the following stronger result.

Theorem A. Let $(S, g)$ be a $(2 n+1)$-dimensional complete Sasaki manifold with a Sasakian structure $\mathcal{S}=\{g, \xi, \eta, \Phi\}$. If $\operatorname{Ric}^{T} \geq \tau g^{T}$ for some constant $\tau>0$, then

$$
\operatorname{diam}(S, g) \leq 2 \pi \sqrt{\frac{2 n-1}{\tau}} .
$$

From Theorem A the result of Hasegawa and Seino follows immediately.

A difficulty which we encounter for our proof of Theorem A is that the positivity of the transverse Ricci curvature does not imply that of the Ricci curvature in general ( $c f$. the identity (2.6)). Therefore we cannot apply Myers' theorem directly to obtain an estimate on the diameter of $(S, g)$. To overcome the difficulty, we consider the variational problem for the energy of horizontal curves in the sense of sub-Riemannian geometry ( $c f$. Section 3). A Sasaki manifold naturally admits the sub-Riemannian structure $\left(D, g^{T}\right)$, and this defines the Carnot-Carathéodory distance for $S$. Since every contact distribution is bracket-generating, the classical theorem of Chow [5] tells us that the Carnot-Carathéodory distance is in fact a distance function on $S$. Moreover, since $D$ satisfies the strong bracket-generating condition, the Hopf-Rinow type theorem holds for the sub-Riemannian structure $\left(D, g^{T}\right)$. This tells us that the Carnot-Carathéodory distance for any two points can be realized as the length of some minimizing horizontal curve through the points, which is called a normal geodesic. We then define the energy functional for horizontal curves and establish the second variational formula for normal geodesics. The second variation of every length-minimizing normal geodesic is always non-negative because every length-minimizing normal geodesic minimizes also the energy functional. By the variational formula and the assumption of the positivity of the transverse Ricci curvature, we can control the length of minimizing normal geodesics, and hence obtain an upper bound on the diameter for the Carnot-Carathéodory distance. Since the Carnot-Carathéodory distance is greater than the Riemannian distance, we obtain the required estimate.

More generally, one can consider a similar problem in the case of a strictly pseudoconvex CR manifold whose Carnot-Carathéodory distances is complete, rather than a complete Sasaki manifold. (For the basic notion of strictly pseudoconvex CR manifolds, see for example [6]) However, our approach depends on the theory of transverse geometry for Riemannian foliations, and this requires the underlying Riemannian metric $g$ to be bundle-like with respect to the Reeb flow. This 
is equivalent to the condition that $g$ is Sasakian, so that our needed ingredients of Riemannian foliation theory are available only for Sasaki manifolds.

This paper is organized as follows: In Section 2 we give a brief review of Sasakian geometry and transverse geometry for Sasaki manifolds. In Section 3 we introduce the notions of Carnot-Carathéodory distance and normal geodesics for a given Sasaki manifold. Then we compute the second variational formula of the energy for normal geodesics on Sasaki manifolds. Finally, in Section 4, we give a proof of Theorem A for complete Sasaki manifolds.

ACKNOWLEDGEMEnTS. The author would like to express his gratitude to Professor Toshiki Mabuchi for valuable comments and suggestions. Many thanks are due also to the referee for his careful reading of the paper and for his numerous suggestions.

\section{Review of Sasakian geometry}

\subsection{Sasaki manifolds}

In this section we review the basic theory of Sasaki manifolds. For the details, see [4]. Throughout this paper, we assume that all manifolds are connected. Let $(S, g)$ be a Riemannian manifold and $(C(S), \bar{g})=\left(S \times \mathbb{R}_{+}, d r^{2}+r^{2} g\right)$ be its cone manifold, where $\mathbb{R}_{+}=\{x \in \mathbb{R} ; x>0\}$ and $r$ is the standard coordinate of $\mathbb{R}_{+}$.

Definition 2.1. $(S, g)$ is called a Sasaki manifold if the cone manifold $(C(S), \bar{g})$ is a Kähler manifold.

A Sasaki manifold $(S, g)$ is often identified with the submanifold $\{r=1\} \subset$ $(C(S), \bar{g})$ and hence the dimension of $S$ is odd. Let $\operatorname{dim} S=2 n+1$. Then, of course, $\operatorname{dim}_{\mathbb{C}} C(S)=n+1$. Let $J$ be a complex structure of the cone $(C(S), \bar{g})$ such that $(\underset{\tilde{\xi}}{C}(S), J, \bar{g})$ is a Kähler manifold, and define $\tilde{\xi}:=J(r \partial / \partial r)$. The restriction $\xi:=\left.\tilde{\xi}\right|_{\{r=1\}}$ of $\tilde{\xi}$ to the submanifold $\{r=1\}$ gives a vector field on $S$. The vector field $\xi$ is called the Reeb vector field. The 1-dimensional foliation $\mathcal{F}_{\xi}$ generated by $\xi$ is called the Reeb foliation. Define a differential 1-form $\eta$ on $S$ by $\eta:=g(\xi, \cdot)$. Then, one can see that

1. $\tilde{\xi}$ is a Killing vector field and satisfies $L_{\tilde{\xi}} J=0$,

2. $\nabla_{\xi} \xi=0$,

3. $\eta(\xi)=1, \iota \xi d \eta=0$,

where $\nabla$ is the Levi-Civita connection of the Riemannian metric $g$. In particular $\xi$ is a Killing vector field on $S$. Since $\eta$ is a non-vanishing 1 -form, it gives a $2 n$ dimensional subbundle $D$ of the tangent bundle $T S$ by

$$
D:=\operatorname{ker} \eta \text {. }
$$


The subbundle $D$ is a contact distribution with the contact form $\eta$, and there is an orthogonal decomposition

$$
T S=D \oplus \mathbb{R} \xi,
$$

where $\mathbb{R} \xi$ is the 1 -dimensional trivial bundle generated by $\xi$.

Next we define a section $\Phi$ of the endomorphism bundle $\operatorname{End}(T S)$ of the tangent bundle $T S$ by $\Phi:=\nabla \xi$. Then it satisfies

$$
\Phi^{2}=-\mathrm{id}+\eta \otimes \xi
$$

and $g(\Phi X, \Phi Y)=g(X, Y)-\eta(X) \eta(Y)$ for each $X, Y \in T S$. In particular, the restriction of $\Phi$ to $D$ (We still denote it by $\Phi$ ) defines a complex structure on $D$. We call the quadruple $\mathcal{S}=\{g, \xi, \eta, \Phi\}$ a Sasakian structure of $S$. The covariant differentiation of $\Phi$ can be written by a language of the curvature;

$$
\left(\nabla_{X} \Phi\right)(Y)=R(X, \xi) Y=g(\xi, Y) X-g(X, Y) \xi
$$

for each $X, Y \in T S$.

\subsection{Transverse geometry on Sasaki manifolds}

Let $(S, g)$ be a Sasaki manifold with a Sasakian structure $\mathcal{S}=\{g, \xi, \eta, \Phi\}$. We can then identify the normal bundle $\nu\left(\mathcal{F}_{\xi}\right)$ of the Reeb foliation $\mathcal{F}_{\xi}$ with $D$. As we described above, $\Phi$ defines a complex structure of $D$. Not only that, $D$ admits a transverse Kähler structure, as described below (for the definition of transverse Kähler structure, see [11]). First note that the Sasaki metric $g$ is bundle-like with respect to the Reeb foliation $\mathcal{F}_{\xi}$ because $\xi$ is a Killing vector field. The restriction $g^{T}:=\left.g\right|_{D \times D}$ of the metric $g$ to $D$ is a Hermitian metric on $D$ whose associated 2 -form is equal to $\left.(1 / 2) d \eta\right|_{D \times D}$;

$$
d \eta(X, Y)=2 g^{T}(\Phi X, Y)
$$

for each $X, Y \in D . g^{T}$ is called the transverse metric. From the transverse metric, we can define a connection on $D$ by

$$
\nabla_{X}^{T} Y:= \begin{cases}\pi_{D}\left(\nabla_{X} Y\right) & \text { if } X \text { is a smooth section of } D, \\ \pi_{D}([X, Y]) & \text { if } X \text { is a smooth section of } \mathbb{R} \xi\end{cases}
$$

where $Y$ is a smooth section of $D$ and $\pi_{D}: T M \rightarrow D$ is the orthogonal projection. One can check easily that this connection is the unique connection on $D$ which is torsion-free and $\nabla^{T} g^{T}=0$. We call it the transverse Levi-Civita connection of $g^{T}$. Furthermore, from (2.2) and (2.4), one can get

$$
\nabla^{T} \Phi=0 .
$$


Hence the triple $\left(D, \Phi, g^{T}\right)$ gives a transverse Kähler structure for the Reeb foliation $\mathcal{F}_{\xi}$ with the transverse Kähler form $\omega^{T}:=\left.(1 / 2) d \eta\right|_{D \times D}$. We can then define the transverse curvature tensor by

$$
\mathrm{R}^{T}(X, Y) Z:=\nabla_{X}^{T} \nabla_{Y}^{T} Z-\nabla_{Y}^{T} \nabla_{X}^{T} Z-\nabla_{[X, Y]}^{T} Z .
$$

Similarly, we can also define the transverse Riemannian curvature tensor $\mathrm{Rm}^{T}$ by

$$
\mathrm{Rm}^{T}(X, Y, Z, W):=g^{T}\left(\mathrm{R}^{T}(X, Y) Z, W\right)
$$

and the transverse Ricci tensor $\operatorname{Ric}^{T}$ by

$$
\operatorname{Ric}^{T}(X, Y):=\sum_{i=1}^{2 n} \operatorname{Rm}^{T}\left(e_{i}, X, Y, e_{i}\right)
$$

for each $X, Y, Z, W \in D$, where $\left\{e_{i}\right\}_{i=1}^{2 n}$ is an orthonormal basis of $D$. Then we have the following formulae for curvatures;

$$
\begin{aligned}
\operatorname{Rm}^{T}(X, Y, Z, W)= & \operatorname{Rm}(X, Y, Z, W)-g(\Phi(X), Z) g(\Phi(Y), W) \\
& +g(\Phi(X), W) g(\Phi(Y), Z)-2 g(\Phi(X), Y) g(\Phi(Z), W), \\
\operatorname{Ric}^{T}(X, Y)= & \operatorname{Ric}(X, Y)+2 g(X, Y)
\end{aligned}
$$

for any $X, Y, Z, W \in D$.

\section{Normal geodesics and the second variational formula}

\subsection{The Carnot-Carathéodory distance}

Let $(S, g)$ be a Sasaki manifold with a Sasakian structure $\mathcal{S}=\{g, \xi, \eta, \Phi\}$. A piecewise smooth curve $\gamma:[0, l] \rightarrow S$ is called horizontal if the differential $\dot{\gamma}(t)$ is tangent to $D_{\gamma(t)}$ for each smooth point $t \in[0, l]$. Given a horizontal curve $\gamma:[0, l] \rightarrow S$, define the length $L_{D}(\gamma)$ of $\gamma$ by

$$
L_{D}(\gamma):=\int_{0}^{l}|\dot{\gamma}(t)| d t .
$$

Then we can define the function $d_{D}: S \times S \rightarrow \mathbb{R}$ by

$$
d_{D}(p, q):=\inf \left\{L_{D}(\gamma) ; \gamma \in \Omega(p, q, D)\right\},
$$

where $p, q \in S$ and $\Omega(p, q, D)$ is the set of all piecewise smooth horizontal curves joining $p$ to $q$. Since the contact distribution $D$ is bracket-generating (i.e., brackets of local sections of $D$ generate all local sections of $T S$ ), the classical theorem of Chow [5] tells us that $\Omega(p, q, D) \neq \emptyset$ for each $p, q \in M$ and hence the function $d_{D}$ is a distance function of $S$. The function $d_{D}$ is called the Carnot-Carathéodory distance for $(S, g)$. It is known that the topology induced by the distance coincides with the original topology of $S$ (see also [10]). For the Riemannian distance function $d$ of $(S, g)$, it is clear that $d \leq d_{D}$. 


\subsection{Normal geodesics}

A notion of normal geodesic is defined in sub-Riemannian geometry as the projection to $S$ of a solution to the "Hamiltonian system", which is described below.

A sub-Riemannian manifold is a triple $\left(S, E, g_{E}\right)$ consisting of a smooth manifold $S$, a subbundle $E$ of the tangent bundle $T S$ and a metric $g_{E}$ on $E$. For a Sasaki manifold $(S, g)$, it is clear that the pair $\left(D, g^{T}\right)$ defines a sub-Riemannian structure of $S$, that is, the triple $\left(S, D, g^{T}\right)$ is a sub-Riemannian manifold. Hence we can apply the notions of sub-Riemannian geometry to Sasakian geometry (the detailed description can be seen in [10] or [14] for example). Let $T^{*} S$ be the cotangent bundle of $S$ and $H_{D}: T^{*} S \rightarrow \mathbb{R}$ be the function on $T^{*} S$ defined by

$$
H_{D}(p, \alpha):=\frac{1}{2}\left(g_{p}^{T}\right)^{-1}\left(\left.\alpha\right|_{D},\left.\alpha\right|_{D}\right)=\frac{1}{2} g_{p}^{-1}(\alpha, \alpha)-\frac{1}{2}\left(\alpha\left(\xi_{p}\right)\right)^{2}
$$

for each $(p, \alpha) \in T^{*} S$. We call the function $H_{D}$ the Hamiltonian function. For any foliation chart $\left(x_{0}, \cdots, x_{2 n}\right)$ with $\xi=\partial / \partial x_{0}$ and the canonical coordinates $\left(x_{0}, \cdots, x_{2 n}, \alpha_{0}, \cdots, \alpha_{2 n}\right)$ on $T^{*} S$, consider the following ordinary differential system:

$$
\left\{\begin{array}{l}
\dot{x}_{i}=\frac{\partial H_{D}}{\partial \alpha_{i}}, \\
\dot{\alpha}_{i}=-\frac{\partial H_{D}}{\partial x_{i}} .
\end{array}\right.
$$

We call it the Hamiltonian system.

Definition 3.1. A smooth curve $\gamma:[0, l] \rightarrow S$ is called a normal geodesic if there exists a cotangent lift $\Gamma(t)=(\gamma(t), \alpha(t)):[0, l] \rightarrow T^{*} S$ which satisfies the Hamiltonian system (3.1).

By existence and uniqueness of solution to an ordinary differential system, the Hamiltonian system (3.1) has unique solution determined by the initial value $\Gamma(0)=(p, \alpha) \in T_{p}^{*} S$. For a normal geodesic $\gamma(t)$ with the cotangent lift $\Gamma(t)=$ $(\gamma(t), \alpha(t))$, the Hamiltonian system can be rewritten as

$$
\left\{\begin{array}{l}
\dot{\gamma}(t)=g^{-1}(\alpha)-\alpha(\xi) \xi, \\
\frac{d \alpha_{i}}{d t}=-\frac{1}{2} \frac{\partial g^{k j}}{\partial x_{i}} \alpha_{k} \alpha_{j},
\end{array}\right.
$$

where $g_{k j}:=g\left(\partial / \partial x_{k}, \partial / \partial x_{j}\right)$ is the component of the Sasaki metric $g$ with respect to the local coordinates $\left(x_{0}, \cdots, x_{2 n}\right)$ and $\left(g^{k j}\right)$ is the inverse matrix of $\left(g_{k j}\right)$. This shows that a normal geodesic is always horizontal. Furthermore, we claim that the system (3.2) implies

$$
\nabla_{\dot{\gamma}(t)} \dot{\gamma}(t)=-2 \alpha_{0} \Phi(\dot{\gamma}(t)),
$$

where $\alpha_{0}=\alpha(\xi)$. First note that $\alpha_{0}$ is constant along the curve $\gamma$. Indeed, the second identity of (3.2) tells us that

$$
\frac{d \alpha_{0}}{d t}=-\frac{1}{2} \frac{\partial g^{k j}}{\partial x_{0}} \alpha_{k} \alpha_{j} .
$$


Since $\xi=\partial / \partial x_{0}$ is a Killing vector field, the right-hand side of (3.4) vanishes.

By the first identity of (3.2), we have

$$
\nabla_{\dot{\gamma}(t)} \dot{\gamma}(t)=\nabla_{\dot{\gamma}(t)}\left(g^{-1}(\alpha(t))-\alpha_{0} \xi\right)=g^{-1}\left(\nabla_{\dot{\gamma}(t)} \alpha(t)\right)-\alpha_{0} \Phi(\dot{\gamma}(t)) .
$$

Hence, to prove our claim, it is sufficient to show $g^{-1}\left(\nabla_{\dot{\gamma}(t)} \alpha(t)\right)=-\alpha_{0} \Phi(\dot{\gamma}(t))$.

Choose a foliation chart $\left(x_{0}, \cdots, x_{2 n}\right)$ with $\xi=\partial / \partial x_{0}$. Then, by the second identity of (3.2), we have

$$
\left(\nabla_{\dot{\gamma}(t)} \alpha(t)\right)\left(\frac{\partial}{\partial x_{j}}\right)=\frac{1}{2} g^{k l}\left(\frac{\partial g_{0 l}}{\partial x_{j}}-\frac{\partial g_{0 j}}{\partial x_{l}}+\frac{\partial g_{j l}}{\partial x_{0}}\right) \alpha_{0} \alpha_{k}
$$

for each $j \in\{0,1, \cdots, 2 n\}$. Since $\xi=\partial / \partial x_{0}$ is a Killing vector field, we have $\partial g_{j l} / \partial x_{0}=0$ and

$$
\frac{\partial g_{0 l}}{\partial x_{j}}-\frac{\partial g_{0 j}}{\partial x_{l}}=g\left(\Phi\left(\frac{\partial}{\partial x_{j}}\right), \frac{\partial}{\partial x_{l}}\right)-g\left(\Phi\left(\frac{\partial}{\partial x_{l}}\right), \frac{\partial}{\partial x_{j}}\right)=-d \eta\left(\frac{\partial}{\partial x_{l}}, \frac{\partial}{\partial x_{j}}\right) .
$$

Hence, by the first identity of (3.2), we obtain

$$
\begin{aligned}
\left(\nabla_{\dot{\gamma}(t)} \alpha(t)\right)\left(\frac{\partial}{\partial x_{j}}\right) & =-\frac{1}{2} g^{k l} d \eta\left(\frac{\partial}{\partial x_{l}}, \frac{\partial}{\partial x_{j}}\right) \alpha_{0} \alpha_{k}=\alpha_{0} g\left(g^{-1}(\alpha(t)), \Phi\left(\frac{\partial}{\partial x_{j}}\right)\right) \\
& =\alpha_{0} g\left(\dot{\gamma}(t), \Phi\left(\frac{\partial}{\partial x_{j}}\right)\right)=-\alpha_{0} g\left(\Phi(\dot{\gamma}(t)), \frac{\partial}{\partial x_{j}}\right) .
\end{aligned}
$$

This proves $g^{-1}\left(\nabla_{\dot{\gamma}(t)} \alpha(t)\right)=-\alpha_{0} \Phi(\dot{\gamma}(t))$ and hence $\nabla_{\dot{\gamma}(t)} \dot{\gamma}(t)=-2 \alpha_{0} \Phi(\dot{\gamma}(t))$, as we claimed.

Note that, for a smooth curve $\gamma:[0, l] \rightarrow S$ which satisfies the equation (3.3) for some $\alpha_{0} \in \mathbb{R}$, we have

$$
\begin{aligned}
\frac{d}{d t}(g(\dot{\gamma}(t), \xi)) & =g\left(\nabla_{\dot{\gamma}(t)} \dot{\gamma}(t), \xi\right)+g\left(\dot{\gamma}(t), \nabla_{\dot{\gamma}(t)} \xi\right) \\
& =-2 \alpha_{0} g(\Phi(\dot{\gamma}(t)), \xi)+g(\dot{\gamma}(t), \Phi(\dot{\gamma}(t))) \\
& =-2 \alpha_{0} g(\Phi(\dot{\gamma}(t)), \xi)+\frac{1}{2} d \eta(\dot{\gamma}(t), \dot{\gamma}(t))=0 .
\end{aligned}
$$

Hence we see that $\gamma$ is horizontal if and only if $\dot{\gamma}(0) \in D_{\gamma(0)}$. Now for each smooth horizontal curve $\gamma:[0, l] \rightarrow S$ which satisfies the equation (3.3), define $\alpha(t):=g\left(\dot{\gamma}(t)+\alpha_{0} \xi\right)$ and $\Gamma(t):=(\gamma(t), \alpha(t))$. Then we can easily check that the curve $\Gamma(t)$ on $T^{*} S$ satisfies (3.1), and hence $\gamma$ is a normal geodesic. This shows the following

Proposition 3.2 (See also [1]). A smooth curve $\gamma:[0, l] \rightarrow S$ is a normal geodesic if and only if it satisfies equation (3.3) for some constant $\alpha_{0} \in \mathbb{R}$ and $\dot{\gamma}(0) \in D_{\gamma(0)}$. 
Here we should remark that a similar description of normal geodesics as solutions to an ordinary differential system is already obtained by Barletta and Dragomir ( $c f$. [1, Theorem 1]) on the more general setting that $S$ is an arbitrary strictly pseudoconvex CR manifold. Though the differential system in [1] is written in terms of the Tanaka-Webster connection rather than the Levi-Civita connection, this coincides with (3.3) if $S$ is a Sasaki manifold.

Remark 3.3. A subbundle $E \subset T S$ of the tangent bundle of $S$ is called strong bracket-generating if for each $p \in S$ and each nonzero local section $X$ of $E$ around $p$ it satisfies $E_{p}+[X, E]_{p}=T_{p} S$. For a Sasaki manifold $(S, g)$, the contact distribution $D$ is always strong bracket-generating. Indeed, for each $p \in S$ and nonzero local section $X$ of $D$ around $p$ we have

$$
\begin{aligned}
g([X, \Phi(X)], \xi) & =g\left(\nabla_{X} \Phi(X), \xi\right)-g\left(\nabla_{\Phi(X)} X, \xi\right) \\
& =-g(\Phi(X), \Phi(X))+g\left(X, \Phi^{2}(X)\right) \\
& =-2 g(X, X) \neq 0 .
\end{aligned}
$$

This shows $\xi_{p} \in D_{p}+[X, D]_{p}$ and hence $T_{p} S=D_{p}+[X, D]_{p}$.

As in the case of Riemannian geometry, it is known that every normal geodesic is locally a unique length-minimizing curve (See [14]). By the fact that $D$ is strong bracket-generating, Strichartz proved that Hopf-Rinow type theorem still holds for the sub-Riemannian manifold $\left(S, D, g^{T}\right)$, i.e., any two points on a Sasaki manifold whose Carnot-Carathéodory distance is complete can be joined by a lengthminimizing normal geodesic (see $[14,15]$ ). The strong bracket-generating condition for $D$ is essential for our proof of Theorem A. Indeed, for a sub-Riemannian manifold $\left(S, D, g_{D}\right)$ such that $D$ is not strong bracket-generating, the Hopf-Rinow type theorem doesn't hold in general. There are some examples of length-minimizing horizontal curves which are not normal geodesics. These examples can be seen in [10].

\subsection{The second variational formula}

For each $p, q \in S$, consider a functional $E_{D}: \Omega(p, q, D) \rightarrow \mathbb{R}$ defined by

$$
E_{D}(\gamma):=\frac{1}{2} \int_{0}^{l} g(\dot{\gamma}(t), \dot{\gamma}(t)) d t,
$$

which is called the energy of horizontal curves. It is well known as in Riemannian geometry, for a constant speed horizontal curve $\gamma$, that $\gamma$ minimizes the length functional $L_{D}: \Omega(p, q, D) \rightarrow \mathbb{R}$ if and only if it minimizes the energy functional. In particular, a length-minimizing normal geodesic is an energy minimizing curve. In this subsection, we shall give the second variational formula of the energy functional for normal geodesics. In this subsection, we assume every curve $\gamma$ is regular, that is, $\gamma$ is smooth and $|\dot{\gamma}(t)| \neq 0$ for all $t \in[0, l]$.

Recall that a variation of a curve $\gamma:[0, l] \rightarrow S$ is a smooth mapping $f$ : $(-\varepsilon, \varepsilon) \times[0, l] \rightarrow S$ which satisfies $f(s, 0)=\gamma(0), f(s, l)=\gamma(l)$ and $f(0, t)=$ 
$\gamma(t)$. A smooth vector field $V$ along $\gamma$ is called a variation vector field of $\gamma$ if it satisfies $V(0)=V(l)=0$. Given a variation $f$ of $\gamma$, we can construct a variation vector field $V$ by $V(t):=\left.(\partial f / \partial s)(s, t)\right|_{s=0}$. Conversely, for each variation vector field $V$ of $\gamma$, there exists a variation $f$ of $\gamma$ whose associated variation vector field is $V$.

For a horizontal curve $\gamma \in \Omega(p, q, D)$, let $f:(-\varepsilon, \varepsilon) \times[0, l] \rightarrow S$ be a variation of $\gamma$. The variation $f$ is said to be admissible if $\partial f / \partial t \in D$ for each $(s, t) \in(-\varepsilon, \varepsilon) \times[0, l]$. Similarly, a variation vector field $V$ of $\gamma$ is said to be admissible if there exists an admissible variation $f$ whose associated variation vector field is $V$. It is easy to see that every admissible variation vector field $V$ of $\gamma$ satisfies

$$
\frac{d}{d t} g(V(t), \xi)=2 g(V(t), \Phi(\dot{\gamma}(t))) \text {. }
$$

Conversely, a similar argument of Ritoré and Rosales in [13] tells us that every variation vector field of $\gamma$ which satisfies the identity (3.6) is always admissible (cf. Appendix A). Hence the set $T_{\gamma} \Omega(p, q, D)$ of all admissible variation vector fields of $\gamma$ is given by

$$
T_{\gamma} \Omega(p, q, D)=\left\{V \in T_{\gamma} \Omega(p, q) ; \frac{d}{d t} g(V(t), \xi)=2 g(V(t), \Phi(\dot{\gamma}(t)))\right\},
$$

where $T_{\gamma} \Omega(p, q)$ is the set of all variation vector fields of $\gamma$.

As in the case of Riemannian geometry, we can easily check that normal geodesics are critical points for the energy functional $E_{D}$. Then we consider the second variation for normal geodesics. The second variational formula for normal geodesics is given by the following:

Proposition 3.4. Let $\gamma:[0, l] \rightarrow S$ be a normal geodesic with the identity (3.3). For each admissible variation $f$ of $\gamma$, define $E_{D}(s):=E_{D}(f(s, \cdot))$ and $V$ to be the associated variation vector field of $f$. Then

$$
\begin{aligned}
E_{D}^{\prime \prime}(0)= & -\int_{0}^{l} g\left(V, \nabla_{\dot{\gamma}(t)} \nabla_{\dot{\gamma}(t)} V+R(V, \dot{\gamma}(t)) \dot{\gamma}(t)\right) d t \\
& +2 \alpha_{0} \int_{0}^{l}\left\{\eta(V) g(V, \dot{\gamma}(t))+g\left(\nabla_{\dot{\gamma}(t)} V, \Phi(V)\right)\right\} d t .
\end{aligned}
$$

Proof. At first, by a standard calculation in Riemannian geometry, we have

$$
\begin{aligned}
E_{D}^{\prime \prime}(s)= & -\int_{0}^{l} g\left(\frac{\partial f}{\partial s}, \frac{D}{d t} \frac{D}{d t} \frac{\partial f}{\partial s}+R\left(\frac{\partial f}{\partial s}, \frac{\partial f}{\partial t}\right) \frac{\partial f}{\partial t}\right) d t \\
& -\int_{0}^{l} g\left(\frac{D}{d s} \frac{\partial f}{\partial s}, \frac{D}{d t} \frac{\partial f}{\partial t}\right) d t .
\end{aligned}
$$

We shall now compute the second term of (3.9). Because $\gamma$ is a normal geodesic, for the integrand we have

$$
\left.g\left(\frac{D}{d s} \frac{\partial f}{\partial s}, \frac{D}{d t} \frac{\partial f}{\partial t}\right)\right|_{s=0}=-\left.2 \alpha_{0} g\left(\frac{D}{d s} \frac{\partial f}{\partial s}, \frac{D}{d t} \xi\right)\right|_{s=0} .
$$


To integrate both sides, notice that

$$
\frac{d^{2}}{d s d t}\left(\eta\left(\frac{\partial f}{\partial s}\right)\right)=2\left\{g\left(\frac{D}{d s} \frac{D}{d t} \xi, \frac{\partial f}{\partial s}\right)+g\left(\frac{D}{d t} \xi, \frac{D}{d s} \frac{\partial f}{\partial s}\right)\right\} .
$$

Furthremore, by (2.2) we have

$$
g\left(\frac{D}{d s} \frac{D}{d t} \xi, \frac{\partial f}{\partial s}\right)=-\eta\left(\frac{\partial f}{\partial s}\right) g\left(\frac{\partial f}{\partial s}, \frac{\partial f}{\partial t}\right)+g\left(\Phi\left(\frac{D}{d t} \frac{\partial f}{\partial s}\right), \frac{\partial f}{\partial s}\right)
$$

and hence

$$
\begin{aligned}
\frac{1}{2} \frac{d^{2}}{d s d t}\left(\eta\left(\frac{\partial f}{\partial s}\right)\right)= & -\eta\left(\frac{\partial f}{\partial s}\right) g\left(\frac{\partial f}{\partial s}, \frac{\partial f}{\partial t}\right)+g\left(\Phi\left(\frac{D}{d t} \frac{\partial f}{\partial s}\right), \frac{\partial f}{\partial s}\right) \\
& +g\left(\frac{D}{d t} \xi, \frac{D}{d s} \frac{\partial f}{\partial s}\right) .
\end{aligned}
$$

Since $(\partial f / \partial s)(s, 0)=(\partial f / \partial s)(s, l)=0$, the integration of both sides of the identity (3.11) with respect to $t$ leads us the following equality;

$$
\int_{0}^{l} g\left(\frac{D}{d t} \xi, \frac{D}{d s} \frac{\partial f}{\partial s}\right) d t=\int_{0}^{l}\left\{\eta\left(\frac{\partial f}{\partial s}\right) g\left(\frac{\partial f}{\partial s}, \frac{\partial f}{\partial t}\right)-g\left(\Phi\left(\frac{D}{d t} \frac{\partial f}{\partial s}\right), \frac{\partial f}{\partial s}\right)\right\} d t .
$$

In particular, by substituting 0 to $s$, we have

$$
\begin{aligned}
\left.\int_{0}^{l} g\left(\frac{D}{d t} \xi, \frac{D}{d s} \frac{\partial f}{\partial s}\right)\right|_{s=0} d t & =\int_{0}^{l} \eta(V) g(V, \dot{\gamma}(t))-g\left(\Phi\left(\nabla_{\dot{\gamma}(t)} V\right), V\right) d t \\
& =\int_{0}^{l} \eta(V) g(V, \dot{\gamma}(t))+g\left(\nabla_{\dot{\gamma}(t)} V, \Phi(V)\right) d t .
\end{aligned}
$$

By combining the identities (3.9), (3.10) and (3.12), we obtain

$$
\begin{aligned}
E_{D}^{\prime \prime}(0)= & -\int_{0}^{l} g\left(V, \nabla_{\dot{\gamma}(t)} \nabla_{\dot{\gamma}(t)} V+R(V, \dot{\gamma}(t)) \dot{\gamma}(t)\right) d t \\
& +2 \alpha_{0} \int_{0}^{l}\left\{\eta(V) g(V, \dot{\gamma}(t))+g\left(\nabla_{\dot{\gamma}(t)} V, \Phi(V)\right)\right\} d t,
\end{aligned}
$$

which is the desired formula.

\section{A proof of Theorem $A$}

In this section we shall give a proof of Theorem A. In order to achieve this, it is sufficient to show the following: 
Theorem 4.1. Let $(S, g)$ be a $(2 n+1)$-dimensional Sasaki manifold with a Sasakian structure $\mathcal{S}=\{g, \xi, \eta, \Phi\}$. If the Carnot-Carathéodory distance is complete and $\operatorname{Ric}^{T} \geq \tau g^{T}$ for some constant $\tau>0$, then

$$
\operatorname{diam}\left(S, d_{D}\right) \leq 2 \pi \sqrt{\frac{2 n-1}{\tau}},
$$

where $\operatorname{diam}\left(S, d_{D}\right):=\sup \left\{d_{D}(p, q) ; p, q \in S\right\}$ is the diameter of $S$ with respect to $d_{D}$.

Theorem A follows immediately from Theorem 4.1 because $d \leq d_{D}$ and the completeness of $(S, g)$ implies that of the Carnot-Carathéodory distance. Our proof of Theorem 4.1 imitates the classical proof of Myers' theorem.

Let $p, q$ be an arbitrary pair of points of $S$ and $\gamma:[0, l] \rightarrow S$ be a lengthminimizing normal geodesic with $\gamma(0)=p, \gamma(l)=q$ and the identity (3.3) (In view of Remark 3.3, such a normal geodesic always exists). We may assume that $|\dot{\gamma}(t)|=1$ for all $t \in[0, l]$. Then $\gamma$ is also a minimizer of $E_{D}: \Omega(p, q, D) \rightarrow \mathbb{R}$ and hence $E_{D}^{\prime \prime}(0) \geq 0$ for each admissible variation of $\gamma$. Choose tangent vectors $X_{1}, \cdots, X_{2(n-1)} \in T_{p} S$ such that $\left\{X_{1}, \cdots, X_{2(n-1)}, \dot{\gamma}(0), \Phi(\dot{\gamma}(0))\right\}$ is an orthonormal basis of $D_{p}$. For each $i \in\{1,2, \cdots, 2(n-1)\}$, consider the following linear differential system for $X_{i}(t) \in D_{\gamma(t)}$ defined by

$$
\nabla_{\dot{\gamma}(t)}^{T} X_{i}(t)=0 \text { and } X_{i}(0)=X_{i}
$$

By existence and uniqueness theorem for linear ordinary differential systems, there is a unique global solution $X_{i}(t) \in D_{\gamma(t)}, t \in[0, l]$.

Lemma 4.2. $\left\{X_{1}(t), \cdots, X_{2(n-1)}(t), \dot{\gamma}(t), \Phi(\dot{\gamma}(t))\right\}$ is an orthonormal basis of $D_{\gamma(t)}$ for all $t \in[0, l]$.

Proof. First note that $g(\dot{\gamma}(t), \dot{\gamma}(t))=g(\Phi(\dot{\gamma}(t)), \Phi(\dot{\gamma}(t)))=1$ and $g(\Phi(\dot{\gamma}(t))$, $\dot{\gamma}(t))=0$. Furthermore, since $g\left(X_{i}, X_{j}\right)=\delta_{i j}$ and

$$
\begin{aligned}
\frac{d}{d t} g\left(X_{i}(t), X_{j}(t)\right) & =g\left(\frac{D}{d t} X_{i}(t), X_{j}(t)\right)+g\left(X_{i}(t), \frac{D}{d t} X_{j}(t)\right) \\
& =g\left(\nabla_{\dot{\gamma}(t)}^{T} X_{i}(t), X_{j}(t)\right)+g\left(X_{i}(t), \nabla_{\dot{\gamma}(t)}^{T} X_{j}(t)\right)=0,
\end{aligned}
$$

we see that $g\left(X_{i}(t), X_{j}(t)\right)=\delta_{i j}$ for each $t \in[0, l]$. Hence it is sufficient to show that $X_{i}(t)$ is perpendicular to both $\dot{\gamma}(t)$ and $\Phi(\dot{\gamma}(t))$.

Choose any $i \in\{1,2, \cdots, 2(n-1)\}$ and define

$$
F_{i}(t):={ }^{t}\left(F_{i, 1}(t), F_{i, 2}(t)\right)={ }^{t}\left(g\left(X_{i}(t), \dot{\gamma}(t)\right), g\left(X_{i}(t), \Phi(\dot{\gamma}(t))\right)\right) .
$$

Then we have

$$
\begin{aligned}
\frac{d F_{i, 1}}{d t} & =\frac{d}{d t} g\left(X_{i}(t), \dot{\gamma}(t)\right)=g\left(\nabla_{\dot{\gamma}(t)} X_{i}(t), \dot{\gamma}(t)\right)+g\left(X_{i}(t), \nabla_{\dot{\gamma}(t)} \dot{\gamma}(t)\right) \\
& =-2 \alpha_{0} g\left(X_{i}(t), \Phi(\dot{\gamma}(t))\right)=-2 \alpha_{0} F_{i, 2}(t),
\end{aligned}
$$


and

$$
\begin{aligned}
\frac{d F_{i, 2}}{d t} & =\frac{d}{d t} g\left(X_{i}(t), \Phi(\dot{\gamma}(t))\right)=g\left(\nabla_{\dot{\gamma}(t)} X_{i}(t), \Phi(\dot{\gamma}(t))\right)+g\left(X_{i}(t), \nabla_{\dot{\gamma}(t)} \Phi(\dot{\gamma}(t))\right) \\
& =2 \alpha_{0} g\left(X_{i}(t), \dot{\gamma}(t)\right)=2 \alpha_{0} F_{i, 1}(t) .
\end{aligned}
$$

Thus the function $F_{i}$ satisfies the following ordinary differential system;

$$
\left\{\begin{aligned}
\frac{d F_{i}}{d t} & =\left(\begin{array}{cc}
0 & -2 \alpha_{0} \\
2 \alpha_{0} & 0
\end{array}\right) F_{i}(t) \\
F_{i}(0) & ={ }^{t}(0,0)
\end{aligned}\right.
$$

This shows that $F_{i}(t)={ }^{t}(0,0)$ for all $t \in[0, l]$ and hence we obtain the desired result.

For each $i \in\{1,2, \cdots, 2(n-1)\}$, define $h(t):=\sin (2 \pi t / l)$ and $V_{i}(t):=h(t) X_{i}(t)$. Since $X_{i}(t)$ is perpendicular to $\Phi(\dot{\gamma}(t))$ for all $t \in[0, l]$, we see that $V_{i} \in$ $T_{\gamma} \Omega(p, q, D)$. Let $f_{i}$ be an admissible variation of $\gamma$ whose variation vector field is $V_{i}$, and define $E_{D, i}(s):=E_{D}\left(f_{i}(s, \cdot)\right)$. Let us calculate the second variation $E_{D, i}^{\prime \prime}(0) \geq 0$ explicitly. Note that since $X_{i}(t) \in D_{\gamma(t)}$ and $\nabla_{\dot{\gamma}(t)}^{T} X_{i}(t)=0$ we have $\eta\left(V_{i}(t)\right)=0$ and

$$
\begin{aligned}
g\left(\nabla_{\dot{\gamma}(t)} V_{i}(t), \Phi\left(V_{i}(t)\right)\right)= & h(t) g\left(h^{\prime}(t) X_{i}(t)+h(t) \nabla_{\dot{\gamma}(t)} X_{i}(t), \Phi\left(X_{i}(t)\right)\right) \\
= & h(t) h^{\prime}(t) g\left(X_{i}(t), \Phi\left(X_{i}(t)\right)\right) \\
& +h^{2}(t) g\left(\nabla_{\dot{\gamma}(t)} X_{i}(t), \Phi\left(X_{i}(t)\right)\right) \\
= & h(t) h^{\prime}(t) d \eta\left(X_{i}(t), X_{i}(t)\right) \\
& +h^{2}(t) g\left(\nabla_{\dot{\gamma}(t)}^{T} X_{i}(t), \Phi\left(X_{i}(t)\right)\right) \\
= & 0 .
\end{aligned}
$$

Hence for $V_{i}$ we have

$$
E_{D, i}^{\prime \prime}(0)=-\int_{0}^{l} g\left(V_{i}, \nabla_{\dot{\gamma}(t)} \nabla_{\dot{\gamma}(t)} V_{i}+R\left(V_{i}, \dot{\gamma}(t)\right) \dot{\gamma}(t)\right) d t
$$

Now we can easily calculate

$$
\begin{aligned}
\nabla_{\dot{\gamma}(t)} \nabla_{\dot{\gamma}(t)} V_{i}(t)= & \nabla_{\dot{\gamma}(t)}\left(h^{\prime}(t) X_{i}(t)+h(t) \nabla_{\dot{\gamma}(t)} X_{i}(t)\right) \\
= & \nabla_{\dot{\gamma}(t)}\left(h^{\prime}(t) X_{i}(t)+h(t) g\left(\nabla_{\dot{\gamma}(t)} X_{i}(t), \xi\right) \xi\right) \\
= & h^{\prime \prime}(t) X_{i}(t)+h^{\prime} g\left(\nabla_{\dot{\gamma}(t)} X_{i}(t), \xi\right) \xi \\
& +\frac{d}{d t}\left(h(t) g\left(\nabla_{\dot{\gamma}(t)} X_{i}(t), \xi\right)\right) \xi+h(t) g\left(\nabla_{\dot{\gamma}(t)} X_{i}(t), \xi\right) \Phi(\dot{\gamma}(t)) .
\end{aligned}
$$


Since $V_{i}(t)$ is perpendicular to both $\xi$ and $\Phi(\dot{\gamma}(t))$, we obtain

$$
g\left(V_{i}(t), \nabla_{\dot{\gamma}(t)} \nabla_{\dot{\gamma}(t)} V_{i}(t)\right)=h(t) h^{\prime \prime}(t)=-\left(\frac{2 \pi}{l}\right)^{2} \sin \left(\frac{2 \pi t}{l}\right) .
$$

Similarly we have

$$
\begin{aligned}
g\left(V_{i}(t), R\left(V_{i}(t), \dot{\gamma}(t)\right) \dot{\gamma}(t)\right) & =\sin ^{2}\left(\frac{2 \pi t}{l}\right) g\left(X_{i}(t), R\left(X_{i}(t), \dot{\gamma}(t)\right) \dot{\gamma}(t)\right) \\
& =\sin ^{2}\left(\frac{2 \pi t}{l}\right) \operatorname{Rm}\left(X_{i}(t), \dot{\gamma}(t), \dot{\gamma}(t), X_{i}(t)\right) \\
& =\sin ^{2}\left(\frac{2 \pi t}{l}\right) \operatorname{Rm}^{T}\left(X_{i}(t), \dot{\gamma}(t), \dot{\gamma}(t), X_{i}(t)\right)
\end{aligned}
$$

by the identity (2.5). By substituting (4.2) and (4.3) in (4.1), we obtain the following inequality;

$0 \leq E_{D, i}^{\prime \prime}(0)=\int_{0}^{l} \sin ^{2}\left(\frac{2 \pi t}{l}\right)\left\{\left(\frac{2 \pi}{l}\right)^{2}-\mathrm{Rm}^{T}\left(X_{i}(t), \dot{\gamma}(t), \dot{\gamma}(t), X_{i}(t)\right)\right\} d t$.

Next we consider

$$
V(t):=h(t) \Phi(\dot{\gamma}(t))+k(t) \xi
$$

for smooth functions $h, k:[0, l] \rightarrow \mathbb{R}$ with $h(0)=h(l)=k(0)=k(l)=0$. Then the condition (3.7) implies that $V \in T_{\gamma} \Omega(p, q, D)$ if and only if $k^{\prime}(t)=2 h(t)$. We put $h(t):=\sin (2 \pi t / l)$ and $k(t):=(l / \pi)(1-\cos (2 \pi t / l))$. Then we can easily check that $k^{\prime}(t)=2 h(t)$. Let $f$ be an admissible variation of $\gamma$ whose variation vector field is $V$, and define $E_{D}(s):=E_{D}(f(s, \cdot))$ At first we have

$$
\begin{aligned}
\nabla_{\dot{\gamma}(t)} V(t)= & \nabla_{\dot{\gamma}(t)} h(t) \Phi(\dot{\gamma}(t))+\nabla_{\dot{\gamma}(t)} k(t) \xi \\
= & h^{\prime}(t) \Phi(\dot{\gamma}(t))+h(t)\left\{\left(\nabla_{\dot{\gamma}(t)} \Phi\right)(\dot{\gamma}(t))+\Phi\left(\nabla_{\dot{\gamma}(t)} \dot{\gamma}(t)\right)\right\} \\
& +k^{\prime}(t) \xi+k(t) \Phi(\dot{\gamma}(t)) \\
= & h^{\prime}(t) \Phi(\dot{\gamma}(t))+h(t)\left(-\xi+2 \alpha_{0} \dot{\gamma}(t)\right)+k^{\prime}(t) \xi+k(t) \Phi(\dot{\gamma}(t)) \\
= & \left(h^{\prime}(t)+k(t)\right) \Phi(\dot{\gamma}(t))+h(t) \xi+2 \alpha_{0} h(t) \dot{\gamma}(t) .
\end{aligned}
$$

In addition, by differentiating again we have

$$
\begin{aligned}
\nabla_{\dot{\gamma}(t)}\left(\left(h^{\prime}(t)+k(t)\right) \Phi(\dot{\gamma}(t))\right)= & \left(h^{\prime \prime}(t)+k^{\prime}(t)\right) \Phi(\dot{\gamma}(t))+\left(h^{\prime}(t)\right. \\
& +k(t)) \nabla_{\dot{\gamma}(t)} \Phi(\dot{\gamma}(t)) \\
= & \left(h^{\prime \prime}(t)+k^{\prime}(t)\right) \Phi(\dot{\gamma}(t)) \\
& +\left(h^{\prime}(t)+k(t)\right)\left(-\xi+2 \alpha_{0} \dot{\gamma}(t)\right) \\
= & \left(h^{\prime \prime}(t)+k^{\prime}(t)\right) \Phi(\dot{\gamma}(t)) \\
& -\left(h^{\prime}(t)+k(t)\right) \xi+2 \alpha_{0}\left(h^{\prime}(t)+k(t)\right) \dot{\gamma}(t),
\end{aligned}
$$


and

$$
\begin{aligned}
\nabla_{\dot{\gamma}(t)} 2 \alpha_{0} h(t) \dot{\gamma}(t) & =2 \alpha_{0}\left(h^{\prime}(t) \dot{\gamma}(t)+h(t) \nabla_{\dot{\gamma}(t) \dot{\gamma}(t))}\right. \\
& =2 \alpha_{0} h^{\prime}(t) \dot{\gamma}(t)-\left(2 \alpha_{0}\right)^{2} h(t) \Phi(\dot{\gamma}(t)) .
\end{aligned}
$$

By combining them we obtain

$$
\begin{aligned}
\nabla_{\dot{\gamma}(t)} \nabla_{\dot{\gamma}(t)} V(t)= & \left(h^{\prime \prime}(t)+3 h(t)-\left(2 \alpha_{0}\right)^{2} h(t)\right) \Phi(\dot{\gamma}(t)) \\
& +2 \alpha_{0}\left(2 h^{\prime}(t)+k(t)\right) \dot{\gamma}(t)-k(t) \xi
\end{aligned}
$$

and hence

$$
g\left(V(t), \nabla_{\dot{\gamma}(t)} \nabla_{\dot{\gamma}(t)} V(t)\right)=h(t)\left(h^{\prime \prime}(t)+3 h(t)-\left(2 \alpha_{0}\right)^{2} h(t)\right)-k^{2}(t) .
$$

For the curvature we have

$$
\begin{aligned}
g(V(t), R(V(t), \dot{\gamma}(t)) \dot{\gamma}(t))= & h^{2}(t) g(\Phi(\dot{\gamma}(t)), R(\Phi(\dot{\gamma}(t)), \dot{\gamma}(t)) \dot{\gamma}(t)) \\
& +2 h(t) k(t) g(\Phi(\dot{\gamma}(t)), R(\xi, \dot{\gamma}(t)) \dot{\gamma}(t)) \\
& +k^{2}(t) g(\xi, R(\xi, \dot{\gamma}(t)) \dot{\gamma}(t)) \\
= & h^{2}(t) \operatorname{Rm}(\Phi(\dot{\gamma}(t)), \dot{\gamma}(t), \dot{\gamma}(t), \Phi(\dot{\gamma}(t))) \\
& +k^{2}(t) \\
= & h^{2}(t) \operatorname{Rm}^{T}(\Phi(\dot{\gamma}(t)), \dot{\gamma}(t), \dot{\gamma}(t), \Phi(\dot{\gamma}(t))) \\
& -3 h^{2}(t)+k^{2}(t) .
\end{aligned}
$$

By substituting (4.5) and (4.6) to (3.8), we obtain

$$
\begin{aligned}
0 \leq & E_{D}^{\prime \prime}(0) \\
= & -\int_{0}^{l}\left\{h(t)\left(h^{\prime \prime}(t)+3 h(t)-\left(2 \alpha_{0}\right)^{2} h(t)\right)-k^{2}(t)\right\} d t \\
& -\int_{0}^{l}\left\{h^{2}(t) \operatorname{Rm}^{T}(\Phi(\dot{\gamma}(t)), \dot{\gamma}(t), \dot{\gamma}(t), \Phi(\dot{\gamma}(t)))-3 h^{2}(t)+k^{2}(t)\right\} d t \\
& -\left(2 \alpha_{0}\right)^{2} \int_{0}^{l} h^{2}(t) d t \\
= & -\int_{0}^{l}\left\{h(t) h^{\prime \prime}(t)+h^{2}(t) \operatorname{Rm}^{T}(\Phi(\dot{\gamma}(t)), \dot{\gamma}(t), \dot{\gamma}(t), \Phi(\dot{\gamma}(t)))\right\} d t \\
= & \int_{0}^{l} \sin ^{2}\left(\frac{2 \pi t}{l}\right)\left\{\left(\frac{2 \pi}{l}\right)^{2}-\operatorname{Rm}^{T}(\Phi(\dot{\gamma}(t)), \dot{\gamma}(t), \dot{\gamma}(t), \Phi(\dot{\gamma}(t)))\right\} d t .
\end{aligned}
$$

Finally, summing (4.4) and (4.7) we obtain

$$
0 \leq \int_{0}^{l} \sin ^{2}\left(\frac{2 \pi t}{l}\right)\left\{\left(\frac{2 \pi}{l}\right)^{2}(2 n-1)-\operatorname{Ric}^{T}(\dot{\gamma}(t), \dot{\gamma}(t))\right\} d t
$$


Furthermore, by assumption $\operatorname{Ric}^{T} \geq \tau g^{T}$,

$$
0 \leq \int_{0}^{l} \sin ^{2}\left(\frac{2 \pi t}{l}\right)\left\{\left(\frac{2 \pi}{l}\right)^{2}(2 n-1)-\tau\right\} d t .
$$

This shows that $0 \leq(2 \pi / l)^{2}(2 n-1)-\tau$ and

$$
d_{D}(p, q)=l \leq 2 \pi \sqrt{\frac{2 n-1}{\tau}} .
$$

Hence we obtain $\operatorname{diam}\left(S, d_{D}\right) \leq 2 \pi \sqrt{(2 n-1) / \tau}$, which completes the proof of Theorem 4.1.

\section{A. Existence of admissible variations}

In this appendix, we shall discuss the existence of admissible variations of regular horizontal curves. Let $\gamma:[0, l] \rightarrow S$ be a regular horizontal curve. For simplicity, we may assume $|\dot{\gamma}(t)|=1$ for each $t \in[0, l]$. Let $V$ be a variation vector field of $\gamma$ which satisfies the equation (3.6). Then we shall show that there exists an admissible variation of $\gamma$ whose associated variation vector field is $V$. Let $\pi$ : $T S \rightarrow S$ be the natural projection. Choose an open interval $\tilde{I}$ containing $[0, l]$ and a smooth map $\tilde{V}: \tilde{I} \rightarrow T S$ so that $\left.\tilde{V}\right|_{[0, l]}=V$. By putting $\tilde{\gamma}(t):=\pi(\tilde{V}(t))$, we have a smooth map $\tilde{\gamma}: \tilde{I} \rightarrow S$ so that $\left.\tilde{\gamma}\right|_{[0, l]}=\gamma$. Then we can define a smooth map $\tilde{f}$ from an open set $U \subset \mathbb{R}^{3}$ containing $\tilde{I} \times\{(0,0)\}$ to $S$ by

$$
\tilde{f}(s, t, u)=\exp _{\exp _{\tilde{\gamma}(t)}(s \tilde{V}(t))}(u \tilde{W}(s, t)),
$$

where $\exp _{p}$ denotes the exponential mapping of $(S, g)$ at $p \in S$ and $\tilde{W}(s, t)$ is the horizontal part of the parallel displacement of $\Phi((d \tilde{\gamma} / d t)(t))$ along the geodesic $s \mapsto \exp _{\tilde{\gamma}(t)}(s \tilde{V}(t))$. It is easy to check that

$$
\tilde{f}(0, t, 0)=\tilde{\gamma}(t), \quad \tilde{f}(s, 0,0)=\gamma(0), \quad \tilde{f}(s, l, 0)=\gamma(l) .
$$

Define a smooth function $\tilde{F}: U \rightarrow \mathbb{R}$ by

$$
\tilde{F}(s, t, u)=g\left(\frac{\partial \tilde{f}}{\partial t}(s, t, u), \xi\right) .
$$

Then, for each $t \in[0, l]$, we have $\tilde{F}(0, t, 0)=g(\dot{\gamma}(t), \xi)=0$ and

$$
\frac{\partial \tilde{F}}{\partial u}(0, t, 0)=-2 \neq 0 \text {. }
$$


By the implicit function theorem, there exists an open neighborhood $R_{t}=\left(-\varepsilon_{t}, \varepsilon_{t}\right) \times$ $\left(t-\varepsilon_{t}, t+\varepsilon_{t}\right)$ of $(0, t) \in \mathbb{R}^{2}$, an open neighborhood $O_{t}$ of $0 \in \mathbb{R}$ and a smooth function $\tilde{\varphi}_{t}: R_{t} \rightarrow O_{t}$ so that $\tilde{\varphi}_{t}(0, t)=0$ and $\tilde{F}\left(s^{\prime}, t^{\prime}, u^{\prime}\right)=0$ if and only if $u^{\prime}=$ $\tilde{\varphi}_{t}\left(s^{\prime}, t^{\prime}\right)$ for any $\left(s^{\prime}, t^{\prime}, u^{\prime}\right) \in R_{t} \times O_{t}$. Note that, for each $t^{\prime} \in\left(t-\varepsilon_{t}, t+\varepsilon_{t}\right) \cap[0, l]$, we have

$$
\tilde{\varphi}_{t}\left(0, t^{\prime}\right)=0
$$

because $\left(0, t^{\prime}, 0\right) \in R_{t} \times O_{t}$ and $\tilde{F}\left(0, t^{\prime}, 0\right)=0$. Moreover, for each $e \in\{0, l\}$ and $s^{\prime} \in\left(-\varepsilon_{e}, \varepsilon_{e}\right)$ we have

$$
\tilde{\varphi}_{e}\left(s^{\prime}, e\right)=0
$$

Indeed, it is easy to see that $\tilde{F}(0, e, 0)=0$ and

$$
\begin{aligned}
\frac{\partial \tilde{F}}{\partial s}(s, e, 0)= & g\left(\frac{D}{d s} \frac{\partial \tilde{f}}{\partial t}(s, e, 0), \xi\right)+g\left(\frac{\partial \tilde{f}}{\partial t}(s, e, 0), \frac{D}{d s} \xi\right) \\
= & \frac{d}{d t} g\left(\frac{\partial \tilde{f}}{\partial s}(s, e, 0), \xi\right)-g\left(\frac{\partial \tilde{f}}{\partial s}(s, e, 0), \frac{D}{d t} \xi\right) \\
& +g\left(\frac{\partial \tilde{f}}{\partial t}(s, e, 0), \Phi\left(\frac{\partial \tilde{f}}{\partial s}(s, e, 0)\right)\right) \\
= & 0
\end{aligned}
$$

for each $s \in\left(-\varepsilon_{e}, \varepsilon_{e}\right)$. This shows $\tilde{F}\left(s^{\prime}, e, 0\right)=0$ and hence $\tilde{\varphi}_{e}\left(s^{\prime}, e\right)=0$. Then, by the uniqueness of the implicit function and (A.2), for each $t_{1}, t_{2} \in[0, l]$ we have $\tilde{\varphi}_{t_{1}}=\tilde{\varphi}_{t_{2}}$ on $R_{t_{1}} \cap R_{t_{2}}$. Hence we can patch them together to obtain a smooth function $\tilde{\varphi}: R \rightarrow \mathbb{R}$, where $R=\cup_{t \in[0, l]} R_{t}$. Since $R$ contains a rectangle $(-\varepsilon, \varepsilon) \times[0, l]$ for sufficiently small $\varepsilon>0$, we can define a smooth map $f$ : $(-\varepsilon, \varepsilon) \times[0, l] \rightarrow S$ by

$$
f(s, t)=\tilde{f}(s, t, \tilde{\varphi}(s, t))
$$

By (A.1), (A.2) and (A.3) we have $f(0, t)=\gamma(t), f(s, 0)=\gamma(0)$ and $f(s, l)=$ $\gamma(l)$. Hence $f$ is a variation of $\gamma$. Since the geodesic $u \mapsto \tilde{f}(s, t, u)$ is horizontal, we have

$$
\begin{aligned}
g\left(\frac{\partial f}{\partial t}(s, t), \xi\right) & =g\left(\frac{\partial \tilde{f}}{\partial t}(s, t, \tilde{\varphi}(s, t)), \xi\right)+\frac{\partial \tilde{\varphi}}{\partial t}(s, t) g\left(\frac{\partial \tilde{f}}{\partial u}(s, t, \tilde{\varphi}(s, t)), \xi\right) \\
& =\tilde{F}(s, t, \tilde{\varphi}(s, t)) \\
& =0
\end{aligned}
$$


for each $(s, t) \in(-\varepsilon, \varepsilon) \times[0, l]$. This shows that the variation $f$ is admissible. Furthermore, for each $t \in[0, l]$ we have

$$
\begin{aligned}
\frac{\partial f}{\partial s}(0, t) & =\frac{\partial \tilde{f}}{\partial s}(0, t, \tilde{\varphi}(0, t))+\frac{\partial \tilde{\varphi}}{\partial s}(0, t) \frac{\partial \tilde{f}}{\partial u}(0, t, \tilde{\varphi}(0, t)) \\
& =\frac{\partial \tilde{f}}{\partial s}(0, t, 0)+\frac{\partial \tilde{\varphi}}{\partial s}(0, t) \frac{\partial \tilde{f}}{\partial u}(0, t, 0) \\
& =V(t)+\frac{\partial \tilde{\varphi}}{\partial s}(0, t) \Phi(\dot{\gamma}(t)) .
\end{aligned}
$$

We shall show that $(\partial \tilde{\varphi} / \partial s)(0, t)=0$. By the identity $\tilde{F}(s, t, \tilde{\varphi}(s, t))=0$, it holds

$$
\frac{\partial \tilde{F}}{\partial s}(0, t, \tilde{\varphi}(0, t))+\frac{\partial \tilde{\varphi}}{\partial s}(0, t) \frac{\partial \tilde{F}}{\partial u}(0, t, \tilde{\varphi}(0, t))=0
$$

Since $\tilde{\varphi}(0, t)=0$, we have

$$
\frac{\partial \tilde{F}}{\partial u}(0, t, \tilde{\varphi}(0, t))=\frac{\partial \tilde{F}}{\partial u}(0, t, 0)=-2
$$

and hence

$$
\frac{\partial \tilde{\varphi}}{\partial s}(0, t)=\frac{1}{2} \frac{\partial \tilde{F}}{\partial s}(0, t, 0)
$$

On the other hand, the identity (3.6) tells us that

$$
\begin{aligned}
\frac{\partial \tilde{F}}{\partial s}(0, t, 0) & =g\left(\frac{D}{d s} \frac{\partial \tilde{f}}{\partial t}(0, t, 0), \xi\right)+g\left(\frac{\partial \tilde{f}}{\partial t}(0, t, 0), \frac{D}{d s} \xi\right) \\
& =g\left(\frac{D}{d t} \frac{\partial \tilde{f}}{\partial s}(0, t, 0), \xi\right)+g(\dot{\gamma}(t), \Phi(V(t))) \\
& =\frac{d}{d t} g\left(\frac{\partial \tilde{f}}{\partial s}(0, t, 0), \xi\right)-g\left(\frac{\partial \tilde{f}}{\partial s}(0, t, 0), \frac{D}{d t} \xi\right)-g(\Phi(\dot{\gamma}(t)), V(t)) \\
& =\frac{d}{d t} g(V(t), \xi)-2 g(V(t), \Phi(\dot{\gamma}(t))) \\
& =0 .
\end{aligned}
$$

This shows that $(\partial \tilde{\varphi} / \partial s)(0, t)=0$ and $(\partial f / \partial s)(0, t)=V(t)$. Hence $f$ is an admissible variation of $\gamma$ whose associated variation vector field is $V$, as required. 


\section{References}

[1] E. BARletta and S. Dragomir, Jacobi fields of the Tanaka-Webster connection on Sasakian manifolds, Kodai Math. J. 29 (2006), 406-454.

[2] A. L. BESSE, "Einstein Manifolds", Springer-Verlag, New York, 1987.

[3] D. E. BLAIR, "Contact Manifolds in Riemannian Geometry", Lecture Notes in Math., Vol. 509, Springer-Verlag, Berlin-Heidelberg-New York, 1976.

[4] C. BOYER and K. GALICKI, "Sasakian geometry", Oxford mathematical monographs, 2008.

[5] W. L. CHOw, Über Systeme von linearen partiellen Differentialgleichungen erster Ordnung, Math. Ann. 117 (1939), 98-105.

[6] S. DRAGOMIR and G. Tomassini, "Differential Geometry and Analysis on CR Manifolds", Progress in Math., Vol. 246, Birkhäuser, Boston-Basel-Berlin, 2006.

[7] M. HARADA, On the curvature of Sasakian manifolds, Bull. Yamagata Univ. Natur. Sci. 7 (1969), 97-106.

[8] M. HARADA, On the minimal diameter of Sasakian manifolds, Bull. Yamagata Univ. Nat. Sci. 7 (1970), 191-203.

[9] I. Hasegawa and M. SEINo, Some remarks on Sasakian geometry-applications of Myers' theorem and the canonical affine connection, J. Hokkaido Univ. Education 32 (1981), 1-7.

[10] R. MONTGOMERY, A tour of subriemannian geometries, their geodesics and applications, Mathematical surveys and monographs, Vol. 91, American Mathematical Society, Providence, RI, 2002.

[11] S. NishiKAWA and P. TONDEUR, Transversal infinitesimal automorphisms for harmonic Kähler foliation, Tohoku Math. J. 40 (1988), 599-611.

[12] Y. NiTTA and K. SEKIYA, A diameter bound for Sasaki manifolds with application to uniqueness for Sasaki-Einstein structure, arXiv:0906.0170v3.

[13] M. RitorÉ and C. RosAles, Area-stationary surfaces in the Heisenberg group $\mathbb{H}^{1}$, Adv. Math. 219 (2008), 633-671.

[14] R. S. STRICHARTZ, Sub-Riemannian geometry, J. Differential Geom. 24 (1986), 221-263.

[15] R. S. S TRICHARTZ, Corrections to "Sub-Riemannian geometry", J. Differential Geom. 30 (1989), 595-596.

[16] S. TANno, The Automorphism groups of almost contact Riemannian manifolds, Tohoku Math. J. 21 (1969), 21-38.

[17] S. TANNO, Sasakian manifolds with constant $\phi$-holomorphic sectional curvature, Tohoku Math. J. 21 (1969), 501-507.

Department of Mathematics

Tokyo Institute of Technology

2-12-1 Ookayama

Meguro-Ku Tokyo 152-8551, Japan

nitta@math.titech.ac.jp 